\title{
A RESOLVENT FOR AN ITERATION METHOD FOR NONLINEAR PARTIAL DIFFERENTIAL EQUATIONS
}

BY

J. W. NEUBERGER(')

\begin{abstract}
Asstract. For each of $m$ and $n$ a positive integer denote by $S(m, i)$ the space of all real-valued symmetric $i$-linear functions on $E_{m}, i=1,2, \ldots, n$. Denote by $L$ a nonzero linear functional on $S(m, n)$, denote by $f$ a real-valued analytic function on $E_{m} \times R \times S(m, 1) \times \cdots \times S(m,[n / 2])$ and denote by $\alpha$ a member of $D(f)$. Denote by $H$ the space of all real-valued functions $U$, analytic at the origin of $E_{m}$, so that $\alpha=\left(0, U(0), U^{\prime}(0), \ldots, U^{(n / 2))}(0)\right)$. For $U \in H, f_{U}(x)=f\left(x, U(x), U^{\prime}(x), \ldots, U^{(n / 2))}(x)\right)$ for all $x$ for which this is defined. A one-parameter semigroup (nonlinear if $f \neq 0) K$ on $H$ is constructed so that if $U \in K$, then $K(\lambda) U$ converges, as $\lambda \rightarrow \infty$, to a solution $Y$ to the partial differential equation $L Y^{(n)}=f_{Y}$. A resolvent $J$ for this semigroup is determined so that $J(\lambda) U$ also converges to $Y$ as $\lambda \rightarrow \infty$ and $s 0$ that $J(\lambda / n)^{n} U$ converges to $K(\lambda) U$ as $n \rightarrow \infty$. The solutions $Y \in H$ of $L Y^{(n)}=f_{Y}$ are precisely the fixed points of the semigroup $K$.
\end{abstract}

1. Introduction. An iteration method for a class of nonlinear partial differential equations was described in [5]. For a given partial differential equation a transformation $T$ was defined which has as its fixed points the solutions to that equation. An iteration method based on $T$ was given. To each function $U$ analytic at the origin of $E_{m}$ a solution to the equation was obtained by means of this iteration. In the present work $T$ is modified slightly to a transformation $S$ so that $S$ has all positive fractional powers $\left\{S^{\lambda}\right\}_{\lambda>0}$. It is shown that if $U$ is a real-valued function which is analytic at the origin of $E_{m}$ then $S^{\lambda} U$ converges as $\lambda \rightarrow \infty$ to a solution to the given partial differential equation-in fact to the same solution obtained by the iteration in [5].

As in the theory of semigroups of nonlinear transformations (cf. [1], [2]), a resolvent for $\left\{S^{\lambda}\right\}_{\lambda>0}$ is found. The rest points of $\left\{S^{\lambda}\right\}_{\lambda>0}$ are precisely the solutions (analytic at the origin of $E_{m}$ ) to the given partial differential equation. Furthermore, every such solution is a rest point of $\left\{S^{\lambda}\right\}_{\lambda>0}$ and given any function $U$ on $E_{m}$ analytic at $0, S^{\lambda} U$ converges to a solution as

Received by the editors July 15, 1975.

AMS (MOS) subject classifications (1970). Primary 47H15, 35 A35.

Key words and phrases. Semigroup of nonlinear transformations, resolvent, nonlinear partial differential equations.

(1) Supported in part by an NSF grant. 
$\lambda \rightarrow \infty$. This observation seems to bring additional nonlinear partial differential equations into the setting of semigroup theory, providing, consequently, new classes of examples for that theory. The constructed resolvent is the basis for several approximation schemes.

In a sense, if $U$ is as above, then $\lim _{\lambda \rightarrow \infty} S^{\lambda} U$ is the nearest solution to the function $U$. This seems to have applicability in the following way: If $U$ is a physically meaningful approximation to a solution to a given partial differential equation, then $S^{\lambda} U$, even for $\lambda$ quite small, may be a better approximation. Instances abound in applied science, of course, in which there is a need for improvement of approximations. It is expected that this comment will be expanded upon in a further work.

2. Statement of main results. Enough preliminary material is given here so that the main results may be stated precisely.

Suppose $n$ is a positive integer. Then $T(E, n)$ denotes the vector space of real-valued $n$-linear functions on the finite dimensional inner product space $E$. For $u \in E$,

$$
\|u\| \equiv\left[\sum_{p_{1}=1}^{m} \cdots \sum_{p_{n}=1}^{m} u\left(e_{p_{1}}, \ldots, e_{p_{n}}\right)^{2}\right]^{1 / 2}
$$

where $m$ is the dimension of $E$ and $e_{1}, \ldots, e_{m}$ is any orthonormal basis of $E$ ([3], [5]). $S(E, n)$ denotes the subspace of $T(E, n)$ consisting of symmetric elements of $T(E, n)$.

If $u \in S(E, p), w \in S(E, k)$, then $u \otimes w$ is the member of $T(E, p+k)$ so that

$$
(u \otimes w)\left(x_{1}, \ldots, x_{p+k}\right)=u\left(x_{1}, \ldots, x_{p}\right) w\left(x_{p+1}, \ldots, x_{p+k}\right)
$$

for all $x_{1}, \ldots, x_{p+k}$ in $E$ and $u \cdot w$ denotes the element of $S(E, p+k)$ nearest (relative to the above norm with $n=p+k$ ) to $u \otimes w$. If $A \in S(E, n), B$ $\in S(E, k), k \geqslant n$, then $A B$ denotes the element of $S(E, k-n)$ so that $\langle A B, C\rangle=\langle B, A \cdot C\rangle$ for all $C \in S(E, k-n)$ where the inner product on the left is in $S(E, k-n)$ and the one on the right is in $S(K, k) . A B$ is then called the inner product of $A$ and $B$.

A function $g$ from an open subset of $E$ to the real numbers $R$ is called analytic at the point $p$ of $E$ (and has radius of convergence at least $r, r>0$ ) provided

$$
g(x)=\sum_{q=0}^{\infty}(1 / q !) g^{(q)}(p)(x-p)^{q}
$$

and 


$$
\sum_{q=0}^{\infty}(1 / q !)\left\|g^{(q)}(p)\right\|\|x-p\|^{q}
$$

converges for all $x$ in $E$ so that $\|x-p\|<r$ where, if $q$ is a positive integer, $g^{(q)}(p) \in S(E, q)$ denotes the $q$ th Fréchet derivative of $g$ at $p$ (cf. [6]). The least upper bound (perhaps infinity) of all such numbers $r$ is denoted by $\rho(g ; p)$ or simply by $\rho(g)$ if $p=0$. This definition of analyticity is equivalent to any of a number of others but the notion of radius of convergence is perhaps a special one.

For $m$ a positive integer, $S\left(E_{m}, n\right)$ is also denoted by $S(m, n)$.

Denote by $H$ the collection of all real-valued functions $U$ on a subset of $E_{m}$ so that

(1) the domain of $U$ is a connected open set containing 0 ,

(2) $U$ is analytic at each point in its domain, and

(3) the domain of $U$ is maximal relative to (1) and (2).

Note that if $U, W \in H$ and $U(x)=W(x)$ for all $x$ in some open subset containing 0 , then $U=W$.

Some notation is now fixed for the remainder of this paper. Denote by each of $m$ and $n$ a positive integer, by $A$ a member of $S(m, n)$ so that $\|A\|=1$ and by $h$ a nonnegative integer $\leqslant n-1$. Denote $E_{m} \times S(m, 0) \times S(m, 1) \times \cdots$ $\times S(m, h)$ by $\Omega(h)(S(m, 0)=R)$. Denote by $\alpha=\alpha_{0}, \alpha_{1}, \ldots, \alpha_{n-1}$ a sequence so that $\alpha_{i} \in S(m, i), i=0,1, \ldots, n-1$. Denote by $H(\alpha)$ the subset of $H$ consisting of all $U$ in $H$ such that $U^{(i)}(0)=\alpha_{i}, i=0,1, \ldots, n-1$. Denote by $f$ a real-valued analytic function with domain an open connected subset (as large as possible) of $\Omega(h)$ which contains $\left(0, \alpha_{0}, \alpha_{1}, \ldots, \alpha_{n}\right)$. If $U \in H(\alpha)$, then $f_{U}$ denotes the member of $H$ so that

$$
f_{U}(x)=f\left(x, U(x), U^{\prime}(x), \ldots, U^{(h)}(x)\right)
$$

for all $x$ for which the right side is defined.

This paper deals mainly with the problem of approximating solutions $U \in H(\alpha)$ to

$$
A U^{(n)}=f_{U}
$$

Following [5], define $T: H(\alpha) \rightarrow H(\alpha)$ so that

$$
\begin{aligned}
(T U)(x)= & \sum_{q=0}^{n-1}(1 / q !) U^{(q)}(0) x^{q} \\
& +\int_{0}^{1}\left((1-j)^{n-1} /(n-1) !\right)\left[U^{(n)}(j x)-\left(A U^{(n)}(j x)-f_{U}(j x)\right) A\right] x^{n}
\end{aligned}
$$

for all $x$ in some region containing $0(j$ is the identity function on the real numbers). 
From Theorem 1 of [5] it follows that if $U \in H(\alpha)$, then $T U=U$ if and only if $A U^{(n)}=f_{U}$.

A sequence $U_{1}, U_{2}, \ldots$ of elements of $H$ is said tu converge to an element $U$ of $H$ if for some $\delta>0$

$$
\lim _{i \rightarrow \infty} \sum_{q=0}^{\infty}(1 / q !)\left\|U_{i}^{(q)}(0)-U^{(q)}(0)\right\| \delta^{q}=0 .
$$

Similarly, if $U_{t} \in H, t>0$ and $U \in H$ then " $U(t) \rightarrow U$ as $t \rightarrow \infty$ " means that for some $\delta>0$

$$
\lim _{t \rightarrow \infty} \sum_{q=0}^{\infty}(1 / q !)\left\|U_{t}^{(q)}(0)-U^{(q)}(0)\right\| \delta^{q}=0 .
$$

THEOREM 1. If $U \in H(\alpha)$, then $\left\{T^{k} U\right\}_{k=1}^{\infty}$ converges to a member $Y$ of $H(\alpha)$ so that $A Y^{(n)}=f_{Y}$.

In [5] for the case $f$ is a polynomial, an iteration method (based on $T$ ) is given which leads to solutions to the equation of Theorem 1 . In the present paper the relatively complicated iteration of [5] is replaced by simply the iteration $\left\{T^{k} W\right\}_{k=1}^{\infty}, W \in H(\alpha)$. Also the requirement that $f$ be a polynomial is replaced by the weaker condition that $f$ be analytic. These improvements are not the main purposes of the present work, however. The main purpose is to give a slight modification $S$ of $T$ so that $S^{\lambda}$ is defined for all $\lambda>0$ in such a way that $S^{t} S^{s}=S^{t+s}$ for all $s, t \geqslant 0$. In this way $\left\{S^{t}\right\}_{t>0}$ becomes a oneparameter semigroup of operators (nonlinear if $f \neq 0$ ) on $H(\alpha)$. As was mentioned above this semigroup has a (nonlinear) resolvent even though the semigroup does not seem to fit any of the usual assumptions. The author hopes that the often observed smoothing properties of resolvents will hold in the present case to the extent that iterations of the type studied here can be extended beyond the set of analytic functions.

Some further notation is required. For $A$ as above and $q$ a positive integer, define $M_{q}, Q_{q}, P_{q}, B_{q}$ as follows:

$$
\begin{aligned}
& M_{q}: S(m, q) \rightarrow S(m, q) \\
& Q_{q}: S(m, q) \rightarrow S(m, q) \\
& P_{q}: S(m, q) \rightarrow S(m, q) \\
& B_{q}: S(m, q-n) \rightarrow S(m, q) \text { so that } \\
& M_{q} w=A(A \cdot w), w \in S(m, q) \\
& Q_{q} w=\left\{\begin{array}{l}
w-A \cdot(A w), w \in S(m, q), q \geqslant n \\
w, q<n
\end{array}\right.
\end{aligned}
$$




$$
\begin{aligned}
& P_{q} w=\left\{\begin{array}{l}
w-A \cdot M_{q-n}^{-1}(A w), w \in S(m, q), q \geqslant n \\
w, q<n
\end{array}\right. \\
& B_{q} w=A \cdot M_{q-n}^{-1} w, w \in S(m, q-n), q \geqslant n .
\end{aligned}
$$

It is noted in [3] and [5] that $Q_{q}^{k} \rightarrow P_{q}$ as $k \rightarrow \infty$ and that $P_{q}$ is an orthogonal projection on $S(m, q), q \geqslant n$. Moreover, $M_{q}$ is positive definite, $q=0,1, \ldots$, $Q_{q}$ nonnegative definite, $q=n, n+1, \ldots$.

For any $\lambda \geqslant 0$, there is a unique nonnegative symmetric power of $Q_{q}$ - written $Q_{q}^{\lambda}$. It holds that $Q_{q}^{\beta} Q_{q}^{\gamma}=Q_{q}^{\beta+\gamma}, \beta, \gamma>0, q=n, n+1, \ldots$

Define $Q$ from $H$ to $H$ so that

$$
(Q U)(x)=\sum_{q=0}^{n-1}(1 / q !) U^{(q)}(0) x^{q}+\sum_{q=n}^{\infty}(1 / q !)\left(Q_{q} U^{(q)}(0)\right) x^{q},\|x\|<\rho(U) .
$$

It is seen that $Q^{\beta} Q^{\gamma}=Q^{\beta+\gamma}, \beta, \gamma>0$.

THEOREM 2. If $\lambda, \delta>0$ then $\left(I-(\lambda / \delta)\left(Q^{\delta}-I\right)\right)^{-1}$ exists and for each $U \in H,\left(I-(\lambda / \delta)\left(Q^{\delta}-I\right)\right)^{-1} U$ converges to an element of $H$ as $\delta \rightarrow 0$.

Denote this limit by $L(\lambda) U$. Denote by $B$ the transformation $H$ to $H$ so that if $U \in H$, then

$$
(B U)(x)=\sum_{q=n}^{\infty}(1 / q !)\left(B_{q} U^{(q-n)}(0)\right) x^{q},
$$

$\|x\|<\rho(U)$. The convergence of this series is assured by Theorem 2 of [5].

TheOREM 3. If $U \in H(\alpha)$ and $\lambda>0$, then there is a unique member $W$ of $H(\alpha)$ so that $W=L(\lambda) U+(I-L(\lambda)) B f_{W}$.

Denote this element $W$ by $J(\lambda) U . J$ is the promised resolvent function indicated in the introductory remarks.

THEOREM 4. If $U \in H(\alpha)$ and $\lambda>0$, there is $W \in H(\alpha)$ so that $J(\lambda / n)^{n} U$ $\rightarrow W$ as $n \rightarrow \infty$.

Denote $W$ by $K(\lambda) U$. Then, moreover,

$$
K(t+s)=K(t) K(s) \text { for all } t, s \geqslant 0 .
$$

THEOREM 5. If $U \in H(\alpha)$ and $h \leqslant n / 2$, there is $Y \in H(\alpha)$ so that

(a) $K(\lambda) U \rightarrow Y$ as $\lambda \rightarrow \infty$,

(b) $T^{k} U \rightarrow Y$ as $k \rightarrow \infty$,

(c) $J(\lambda) U \rightarrow Y$ as $\lambda \rightarrow \infty$.

Moreover, $A Y^{(n)}=f_{Y}$. 
Following [5], denote by $H_{0}$ the range of $B$. It may be seen that each member of $H(\alpha) / H_{0}$ contains just one solution to the equation in Theorem 5 . Moreover, if $\lambda>0$ and $k$ is a positive integer, then each of $K(\lambda), J(\lambda)$ and $T^{k}$ leaves each member of $H(\alpha) / H_{0}$ invariant. So, various of the iterations described' above are carried out entirely within cosets of $H(\alpha) / H_{0}$ (for given choice of $U \in H(\alpha)$ ).

The "slight modification $S$ " of $T$ mentioned early in the introduction is defined by $S=K(1)$.

It is remarked that the convergence in Theorem 3 rests upon the following from [4]:

If each $n$ and $k$ is a positive integer, $A \in S(m, n)$ and $B \in S(m, k)$, then

$$
\|A \cdot B\|^{2}\left(\begin{array}{c}
n+k \\
n
\end{array}\right) \geqslant\|A\|^{2}\|B\|^{2} .
$$

\section{Some preliminary results.}

Proof of Theorem 2. Suppose $q \in Z^{+}, q \geqslant n$ and $\lambda>0$. If $w \in S(m, q)$, $w \neq 0$ and $\beta$ is a number so that $Q_{q} w=\beta w$, then $0 \leqslant \beta \leqslant 1$ and $Q_{q}^{\delta} w$ $=\beta^{\delta} w$. If $\beta>0$, then

$$
\begin{aligned}
\left(I-(\lambda / \delta)\left(Q_{q}^{\delta}-I\right)\right)^{-1} w & =\left(1-(\lambda / \delta)\left(\beta^{\delta}-1\right)\right)^{-1} w \\
& \rightarrow(1-\lambda \ln \beta)^{-1} w \text { as } \delta \rightarrow 0 .
\end{aligned}
$$

If $\beta=0$, then $\left(I-(\lambda / \delta)\left(Q_{q}^{\delta}-I\right)\right)^{-1} w=(1+(\lambda / \delta))^{-1} w \rightarrow 0$ as $\delta \rightarrow 0$.

It should be noted that if $\delta>0$, then $\left|Q^{\delta}\right| \leqslant 1,\left|\left(I-(\lambda / \delta)\left(Q_{q}^{\delta}-I\right)\right)^{-1}\right|$ $\leqslant 1$.

Since $Q_{q}$ is nonnegative and symmetric, $S(m, q)$ has a basis of eigenvectors of $Q_{q}$ and so $\lim _{\delta \rightarrow 0}\left(I-(\lambda / \delta)\left(Q_{q}^{\delta}-I\right)\right)^{-1}$ exists and is a linear transformation (which must also have norm not exceeding one) from $S(m, q)$ to $S(m, q)$.

If $q \in Z^{+}, q<n$, then $\left(I-(\lambda / \delta)\left(Q_{q}^{\delta}-I\right)\right)^{-1}=I$ for all $\delta>0$, so of course

$$
\lim _{\delta \rightarrow 0}\left(I-(\lambda / \delta)\left(Q_{q}^{\delta}-I\right)\right)^{-1}=I .
$$

Denote $\lim _{\delta \rightarrow 0}\left(I-(\lambda / \delta)\left(Q_{q}^{\delta}-I\right)\right)^{-1}$ by $L(\lambda)_{q}$ and, for $U \in H$, define $L(\lambda) U \in H$ so that

$$
(L(\lambda) U)(x)=\sum_{q=0}^{\infty}(1 / q !)\left(L(\lambda)_{q} U^{(q)}(0)\right) x^{q}, \quad\|x\|<\rho(U) .
$$

It is easy to check that if $\delta>0$, then $\left(I-(\lambda / \delta)\left(Q^{\delta}-I\right)\right)^{-1}$ exists and $\lim _{\delta \rightarrow 0}\left(I-(\lambda / \delta)\left(Q^{\delta}-I\right)\right)^{-1}=L(\lambda) U$ for each $U \in H$.

The following is needed for Theorem 3. 
LEMMA 1. Suppose $t$ is a positive integer, each of $E, \Omega_{1}, \ldots, \Omega_{t}$ is a finite dimensional inner product space and $\Omega=\Omega_{1} \times \cdots \times \Omega_{t}$. Suppose furthermore that $f$ is a real-analytic function with domain an open subset of $\Omega$ and that $\beta$ is $a$ point of $\Omega$. Then there is a real-valued analytic function $F$ on an open subset of $E_{t}$ so that

(1) if $\phi$ is an analytic function from an open subset of $E, 0$ is in $D(\phi), \phi(0)=\beta$ and $R(\phi) \subset D(f)$, then for each positive integer $q$,

$$
\left\|(f(\phi))^{(q)}(0)\right\| \leqslant(F(\Phi))^{(q)}(0)
$$

where

$$
\Phi=\left(\Phi_{1}, \ldots, \Phi_{t}\right), \quad \Phi_{i}(s)=\sum_{q=0}^{\infty}(1 / q !)\left\|\phi_{i}^{(q)}(0)\right\| s^{i}, \quad i=1, \ldots, t,
$$

for all s such that $|s|<\inf \rho\left(\phi_{1}\right), \ldots, \rho\left(\phi_{t}\right), \phi=\left(\phi_{1}, \ldots, \phi_{t}\right)$, and

(2) $\left\|f^{(q)}(\beta)\right\|=\left\|F^{(q)}(\gamma)\right\|$ where $\beta=\left(\beta_{1}, \ldots, \beta_{t}\right)$ and $\gamma=\left(\left\|\beta_{1}\right\|, \ldots\right.$, $\left.\left\|\beta_{t}\right\|\right)$.

Proof of Lemma 1. Using a formula for the derivative of the composition of two functions on normed linear spaces (cf. [5, Proposition 5]) one has that if $q$ is a positive integer and $x=x_{1}, \ldots, x_{q}$ is a sequence of which each element is in $E$, then

$$
(f(\phi))^{(q)}(0) x=\sum_{p \in S_{q}} f^{(|p|)}(\beta)\left(\phi^{\left(\left|p_{1}\right|\right)}(0) x\left(p_{1}\right), \ldots, \phi^{\left(\left|p_{|p|}\right|\right)}(0) x\left(p_{|p|}\right)\right)
$$

where $S_{q}$ is the set of all partitions of $\{1, \ldots, q\}$, if $p \in S_{q}$ then $|p|$ denotes the number of elements in $p$ and $p_{1}, \ldots, p_{|p|}$ denotes the enumeration of $p$ so that if $1 \leqslant i<k \leqslant|p|$, then the least element of $p_{i}$ is less than the least element of $p_{k}$ and $\phi^{\left(\left|p_{i}\right|\right)}(0) x\left(p_{i}\right)$ denotes $\phi^{\left(\left|p_{i}\right|\right)}(0)\left(x_{q_{1}}, \ldots, x_{q_{s}}\right)$ where $p_{i}=q_{1}, \ldots, q_{s}$. If $k \in Z^{+}$, then $\Gamma(t, k)$ denotes the set of all sequences $r_{1}, \ldots, r_{k}$ such that $r_{i} \in\{1, \ldots, t\}, i=1, \ldots, k$. Then

$$
\begin{aligned}
(f(\phi))^{(q)}(0) x & \\
& =\sum_{p \in S_{q}} \sum_{r \in \Gamma(t,|p|)} f^{(|p|)}(\beta)\left(\left(\phi^{\left(\left|p_{1}\right|\right)}(0) x\left(p_{1}\right)\right)_{\eta}, \ldots,\left(\phi^{\left(\left|p_{|p|}\right|\right)}(0) x\left(p_{|p|}\right)\right)_{r|p|}\right) \\
& =\sum_{p \in S_{q}} \sum_{r \in \Gamma^{\prime}(t,|p|)}\left(f^{(|p|)}(\beta)\right) r\left(\phi_{\eta}^{\left(\left|p_{1}\right|\right)}(0) x\left(p_{1}\right), \ldots, \phi_{\eta_{|p|}}^{\left(\left|p_{|p|}\right|\right)}(0) x\left(p_{|p|}\right)\right)
\end{aligned}
$$

where if $r \in \Gamma(t,|p|), p \in S_{q}$ and $i \in\{1, \ldots,|p|\}$, then (i) $\left(\phi^{\left(\left|p_{i}\right|\right)}(0) x\left(p_{i}\right)\right)_{\eta}$ denotes the member of $\Omega$ with all components other than the $r_{\text {th }}$ one 0 and with the $r_{i}$ th component equal to the $r_{i}$ th component of $\phi^{\left(\left|p_{i}\right|\right.}(0) x\left(p_{i}\right)$, (ii) 
$\phi_{\eta_{i}}^{\left(\left|p_{i}\right|\right)}(0) x\left(p_{i}\right)$ denotes the $r_{i}^{\text {th }}$ component of $p^{\left(\left|p_{i}\right|\right)}(0) x\left(p_{i}\right)$, and (iii) $\left(f^{(|p|)}(\beta)\right)$, denotes the multilinear function on $\Omega_{\eta} \times \cdots \times \Omega_{\eta_{n}}$ so that if $y_{i} \in \Omega_{\eta}, z_{i} \in \Omega,\left(z_{i}\right)_{\delta}=0, \delta \neq r_{i},\left(z_{i}\right)_{\eta}=y_{i}, i=1, \ldots,|p|$, then

$$
\left(f^{(|p|)}(\beta)\right)\left(z_{1}, \ldots, z_{|p|}\right)=\left(f^{(|p|)}(\beta)\right)_{r}\left(y_{1}, \ldots, y_{|p|}\right) .
$$

More simply said, $\left(f^{(|p|)}(\beta)\right)_{r}$ is the $r$-partial derivative of $f$ at $\beta, r \in \Gamma(t,|p|)$.

For $p \in S_{q}, r \in \Gamma(t,|p|), e_{1}, \ldots, e_{m}$ an orthonormal basis of $E_{m}$, define $L_{p, r}$ on $E^{q}$ so that if $x=x_{1}, \ldots, x_{q} \in E$, then

$$
L_{p, r} x=\left(f^{(|p|)}(\beta)\right)_{r}\left(\phi_{\eta}^{\left(\left|p_{1}\right|\right)}(0) x\left(p_{1}\right), \ldots, \phi_{\eta_{1}}^{\left(\left|p_{p \mid}\right|\right)}(0) x\left(p_{|p|}\right)\right) .
$$

Then

$$
\begin{aligned}
\left\|L_{p, r}\right\|^{2} & =\sum_{s \in \Gamma(m, q)}\left(L_{p, r} e_{s}\right)^{2} \\
& \leqslant\left\|\left(f^{(|p|)}(\beta)\right)_{r}\right\|^{2} \sum_{s \in \Gamma(m, q)}\left\|\phi_{\eta}^{\left(\left|p_{1}\right|\right)}(0) e_{s}\left(p_{1}\right)\right\|^{2} \cdots\left\|\phi_{\eta_{A}}^{\left(\left|p_{1}\right|\right)}(0) e_{s}\left(p_{|p|}\right)\right\|^{2} \\
& =\left\|\left(f^{(|p|)}(\beta)\right)_{r}\right\|^{2}\left\|\phi_{\eta}^{\left(\left|p_{1}\right|\right)}(0)\right\|^{2} \cdots\left\|\phi_{\eta_{A}}^{\left(\left|p_{1, p}\right|\right)}(0)\right\|^{2} .
\end{aligned}
$$

Hence

$$
\begin{aligned}
\left\|(f(\phi))^{(q)}(0)\right\| & \leqslant \sum_{p \in S_{q}} \sum_{r \in \Gamma(t,|p|)}\left\|\left(f^{(|p|)}(\beta)\right)_{r}\right\|\left\|_{\eta}^{\left(\left|p_{1}\right|\right)}(0)\right\| \cdots\left\|\phi_{\eta_{d}}^{(|p|)}(0)\right\| \\
& \leqslant \sum_{p \in S_{q}} \sum_{r \in \Gamma(t,|p|)}\left(F^{(|p|)}(\Phi(0))\right)_{r} \Phi_{\eta}^{(|p|)}(0) \cdots \Phi_{\eta_{A}}^{\left(\left|p_{\mid}\right| A^{\prime}\right)}(0) \\
& =(F(\Phi))^{(q)}(0)
\end{aligned}
$$

where $F$ is described as follows: Denote $\left(\left\|\beta_{1}\right\|, \ldots,\left\|\beta_{l}\right\|\right)$ by $\gamma$ and define, for $i$ a nonnegative integer, $\delta_{l} \in S\left(E_{t}, i\right)$ by

$$
\delta_{i}\left(y_{1}, \ldots, y_{i}\right)=\sum_{r \in \Gamma(t, i)} y_{1, n} \cdots y_{i, r_{i}} \varepsilon(i ; r)
$$

for each of $y_{1}, \ldots, y_{i} \in E_{t}$ where $\varepsilon(i ; r) \equiv\left\|\left(f^{(i)}(\beta)\right)_{r}\right\|$. Then take $F(y)$ $=\sum_{i=0}^{\infty}(1 / i !) \delta_{i}(y-\gamma)^{i}$ for all $y \in E_{t}$ such that $\|y-\gamma\|<\rho(f)$. This completes the definition of $F$.

Note that if $i$ is a nonnegative integer,

$$
\left\|f^{(i)}(\beta)\right\|^{2}=\sum_{r \in \Gamma(t, i)} \varepsilon(i ; r)^{2}=\left\|\delta_{i}\right\|^{2}=\left\|F^{(i)}(\gamma)\right\|^{2}
$$

since $F^{(i)}(\gamma)=\delta_{i}, i=0,1,2, \ldots$ 
Proof of Theorem 3. Suppose $U \in H(\alpha)$ and $\lambda>0$. Define $\Omega_{1}=E_{m}, \Omega_{2}$ $=R, \Omega_{i}=S(m, i-2), i=3,4, \ldots, h+2$ and denote $\Omega_{1} \times \cdots \times \Omega_{h+2}$ by $\boldsymbol{\Omega}$.

Denote by $\left\{w_{i}\right\}_{i=0}^{\infty}$ the sequence so that

(a) $w_{i} \in S(m, i), i=0,1,2, \ldots$,

(b) $\left(w_{0}, w_{1}, \ldots, w_{n-1}\right)=\alpha$, and then

(c) if $q$ is a positive integer $\geqslant n-1$ and $V_{q}(x)=\sum_{i=0}^{q}(1 / i !) w_{i} x^{l}, x \in E_{m}$,

$$
w_{q+1}=\left(L(\lambda)_{q} U\right)^{(q)}(0)+\left(I-L(\lambda)_{q}\right) B_{q} f_{V}^{(q-n)}(0) .
$$

One may think of obtaining $w_{n}, w_{n+1}, \ldots$, by assuming there is $W \in H(\alpha)$ so that $W=L(\lambda) U+(I-L(\lambda)) B f_{W}$ and then, inductively, defining

$$
w_{q}=W^{(q)}(0)=L(\lambda)_{q} U^{(q)}(0)+\left(I-L(\lambda)_{q}\right) B_{q} f_{W}^{(q-n)}(0)
$$

obtaining $w_{q}$ in terms of $w_{0}, w_{1}, \ldots, w_{q-1}$.

The theorem will be demonstrated if it can be shown that there is $\delta>0$ so that $\sum_{q=0}^{\infty}(1 / q !)\left\|w_{q}\right\| \delta^{q}$ converges since then $W$ can be chosen to be that member of $H(\alpha)$ so that

$$
W(x)=\sum_{q=0}^{\infty}(1 / q !) w_{q} x^{q}, \quad\|x\|<\delta .
$$

To begin to accomplish this, denote by $F$ the function which relates to $f$ as in the preceding lemma (here $t=h+2$ ).

Start with a calculation of the norm of $\left(I-L(\lambda)_{q}\right) B_{q}, q \in Z^{+}, q \geqslant n$. For this it is shown first that

$$
\left(I-G_{q}\right)^{\delta} A \cdot z=A \cdot\left(I-M_{q-n}\right)^{\delta} z
$$

for $z \in S(m, q-n), \delta>0$.

Suppose $M_{q-n} w=\varepsilon w$ for some nonzero member $w$ of $S(m, q-n)$ and some number $\varepsilon$ (necessarily in $(0,1])$. Then

$$
\begin{gathered}
A(A \cdot w)=\varepsilon w, \quad A \cdot(A(A \cdot w))=\varepsilon A \cdot w, \quad G_{q}(A \cdot w)=\varepsilon A \cdot w, \\
\left(I-G_{q}\right)(A \cdot w)=(1-\varepsilon)(A \cdot w), \quad\left(I-G_{q}\right)^{8}(A \cdot w)=(1-\varepsilon)^{8}(A \cdot w),
\end{gathered}
$$

but also

$$
\begin{aligned}
\left(I-M_{q-n}\right) w & =(1-\varepsilon) w, \quad\left(I-M_{q-n}\right)^{\delta} w=(1-\varepsilon)^{\delta} w \\
A \cdot\left(I-M_{q-n}\right)^{\delta} w & =(1-\varepsilon)^{\delta} A \cdot w \quad \text { so } A \cdot\left(I-M_{q-n}\right)^{\delta} w=\left(I-G_{q}\right)^{\delta}(A \cdot w) .
\end{aligned}
$$


But $S(m, q-n)$ is spanned by eigenvectors of $M_{q-n}$. Hence by linearity, $A \cdot\left(I-M_{q-n}\right)^{\delta} z=\left(I-G_{q}\right)^{\delta}(A \cdot z)$ for all $z \in S(m, q-n)$. From this it follows that if $z \in S(m, q-n)$,

$$
\begin{aligned}
\left(I-L(\lambda)_{q}\right) A \cdot M_{q-n}^{-1} z & =\lim _{\delta \rightarrow 0}\left(I-\left(I-(\lambda / \delta)\left(\left(I-G_{q}\right)^{\delta}-I\right)\right)^{-1} A \cdot M_{q-n}^{-1}\right) z \\
& =\lim _{\delta \rightarrow 0} A \cdot M_{q-n}^{-1}\left(I-\left(I-(\lambda / \delta)\left(\left(I-M_{q-n}\right)^{\delta}-I\right)\right)^{-1}\right) z .
\end{aligned}
$$

If $w$ is an eigenvector of $M_{q-n}^{-1}$ with eigenvalue $\varepsilon$, then

$$
\begin{aligned}
\lim _{\delta \rightarrow 0}(I- & \left.(\lambda / \delta)\left(\left(I-M_{q-n}\right)^{\delta}-I\right)\right)^{-1} w \\
\quad & =\lim _{\delta \rightarrow 0}\left(1-\left(\lambda / \hat{\delta} ;\left((1-\varepsilon)^{\delta}-1\right)\right)^{-1} w\right. \\
& =d_{\lambda, \varepsilon} w= \begin{cases}(1-\lambda \ln (1-\varepsilon))^{-1} & \text { if } 0<\varepsilon \leqslant 1, \\
0 & \text { if } \varepsilon=1 .\end{cases}
\end{aligned}
$$

Hence

$$
\begin{aligned}
\left\|\left(I-L(\lambda)_{q}\right) B_{q} w\right\|^{2} & =\lim _{\delta \rightarrow 0}\left\|A \cdot M_{q-n}^{-1}\left(I-\left(I-(\lambda / \delta)\left(\left(I-M_{q-n}\right)^{\delta}-I\right)\right)^{-1}\right) w\right\| \\
& =\lim _{\delta \rightarrow 0}\left\langle M_{q-n}^{-1}\left(I-\left(I-(\lambda / \delta)\left(\left(I-M_{q-n}\right)^{\delta}-I\right)\right)^{-1}\right)^{2} w, w\right\rangle \\
& =\left\langle\varepsilon^{-1}\left(1-d_{\lambda, \varepsilon}\right)^{2} w, w\right\rangle=\varepsilon^{-1}\left(1-d_{\lambda, \varepsilon}\right)^{2}\|w\|^{2} .
\end{aligned}
$$

Hence $\left|\left(I-L(\lambda)_{q}\right) B_{q}\right|^{2} \leqslant \sup _{0<e<1} \varepsilon^{-1}\left(1-d_{\lambda, e}\right)^{2} \equiv b_{\lambda}^{2}$. This last upper bound exists since $\lim _{e \rightarrow 0} \varepsilon^{-1}\left(1-d_{\lambda, e}\right)^{2}=0$ and $d_{\lambda, e}$ is continuous in $\varepsilon$ on $(0$, 1]. It is emphasized that this estimate

$$
\left|\left(I-L(\lambda)_{q}\right) B_{q}\right|^{2} \leqslant b_{\lambda}^{2}
$$

holds for $q=n, n+\mathrm{i}, \ldots$.

Denote by $g$ the real-valued analytic function

$$
g^{(q)}(0)=\left\|L(\lambda)_{q} U^{(q)}(0)\right\|, \quad q=0,1,2, \ldots
$$

Denote by $\delta$ a small enough positive number so that there is a unique function $y$ on $(-\delta, \delta)$ so that $\left(y(0), y^{\prime}(0), \ldots, y^{(n-1)}(0)\right)=(\|U(0)\|$, $\left.\left\|U^{\prime}(0)\right\|, \ldots,\left\|U^{(n-1)}(0)\right\|\right)$ and

$$
y^{(n)}(x)=g(x)+b_{\lambda} F\left(x, y(x), y^{\prime}(x), \ldots, y^{(h)}(x)\right), \quad|x|<\delta .
$$

That there is such a positive number $\delta$ and such a function $y$ follows from basic existence and rmiqueness theory for ordinary differential equations. 
It follows by induction that $\left\|w_{q}\right\| \leqslant y^{(q)}(0), q=0,1,2, \ldots$ since if $q$ is a positive integer, $W \in H(\alpha)$ and $\left\|W^{(i)}(0)\right\| \nLeftarrow y^{(i)}(0), i=0,1, \ldots, q-1$, then

$$
\begin{aligned}
\|(L(\lambda) U+(I- & \left.L(\lambda)) B f_{W}\right)^{(q)}(0) \| \\
& \leqslant\left\|(L(\lambda) U)^{(q)}(0)\right\|+\left\|\left(I-L(\lambda)_{q}\right) B_{q} f_{W}^{(q-n)}(0)\right\| \\
& \leqslant g^{(q)}(0)+b_{\lambda}\left(F\left(j, y, \ldots, y^{(h)}\right)\right)^{q-n}(0)
\end{aligned}
$$

using Lemma $1(j(t)=t$ for all $t \in R)$.

Since there is a number $\delta^{\prime}, 0<\delta^{\prime} \leqslant \delta$ so that if $|t|<\delta^{\prime}$, then $\sum_{i=0}^{\infty}(1 / i !) y^{(i)}(0) t^{i}$ converges, it follows that $\sum_{q=0}^{\infty}(1 / q !)\left\|w_{q}\right\|\|x\|^{q}$ converges and hence $\sum_{q=0}^{\infty}(1 / q !) w_{q} x^{q}$ converges if $\|x\|<\delta^{\prime}$.

Define now $W \in H(\alpha)$ so that $W(x)=\sum_{q=0}^{\infty}(1 / q !) w_{q} x^{q},\|x\|<\delta^{\prime}$. From the way $\left\{w_{q}\right\}_{q=0}^{\infty}$ was defined it is seen that $W=L(\lambda) U+(I-L(\lambda)) B f_{W}$. This completes a proof of Theorem 3.

Denote $W$ by $J(\lambda) U$.

Lemma 2. If $\lambda_{0}>0, q \in Z^{+}, w \in S(m, q)$ and $\varepsilon>0$, then there is $\delta>0$ so that if $0 \leqslant \lambda \leqslant \lambda_{0}, k \in Z^{+}, \delta>t_{i} \geqslant 0, i=1, \ldots, k$ and $t_{1}+\cdots+t_{k}$ $=\lambda$, then

$$
\left\|L\left(t_{k}\right)_{q} \cdots L\left(t_{1}\right)_{q} w-\left(I-G_{q}\right)^{\lambda} w\right\|<\varepsilon .
$$

Proof. Clearly this follows if it can be shown in case $w$ an eigenvector of $G$. Suppose $w \in S(m, q), w \neq 0$, and $G w=\theta w$. Then $0 \leqslant \theta \leqslant 1$. As in the proof of Theorem 3,

$$
\begin{aligned}
L(t)_{q} w & =\lim _{\delta \rightarrow 0}\left(I-(t / \delta)\left(\left(I-G_{q}\right)^{\delta}-I\right)\right)^{-1} w \\
& =\lim _{\delta \rightarrow 0}\left(1-(t / \delta)\left((1-\theta)^{\delta}-1\right)\right)^{-1} w \\
& = \begin{cases}0 \cdot w & \text { if } \theta=1 \\
(1-t \ln (1-\theta))^{-1} w & \text { if } 0 \leqslant \theta \leqslant 1 .\end{cases}
\end{aligned}
$$

So if $\theta=1, L\left(t_{k}\right)_{q} \cdots L\left(t_{1}\right)_{q} w=0 \cdot w$ and $\left(I-G_{q}\right)^{\lambda} w=0$. For $0 \leqslant \theta<1$, $t_{1}+\cdots+t_{k}=\lambda$,

$$
L\left(t_{k}\right) \cdots L\left(t_{1}\right) w=\left(1-t_{k} \ln (1-\theta)\right)^{-1} \cdots\left(1-t_{1} \ln (1-\theta)\right)^{-1} w
$$

and $\left(I-G_{q}\right)^{\lambda} w=(1-\theta)^{\lambda} w$.

The fact that $\lim _{n \rightarrow \infty}(1-(\lambda / n) \ln (1-\theta))^{-n}=e^{\lambda \ln (1-\theta)}=(1-\theta)^{\lambda}$ is familiar. Small modifications of an argument for this fact give the necessary fact for the lemma. 
LEMMA 3. If $q \in Z^{+}$and $V_{q}(t) \equiv\left(I-G_{q}\right)^{t}, t \geqslant 0$, then $V_{q}$ is of bounded variation on all of $[0, \infty)$.

Proof. If $w$ is an eigenvector of $G_{q}$ with eigenvalue $\theta$, then

$$
V_{q}(t) w=(1-\theta)^{t} w, \quad t \geqslant 0
$$

If $\theta=1, V_{q}(\cdot) w$ is constant at 0 ; if $0 \leqslant \theta<1$, then $V_{q}(\cdot) w$ has total variation $\|w\|$ on $[0, \infty)$. Since a finite set of eigenvectors of $G_{q}$ spans $S(m, q)$, the result follows.

LEMMA 4. If $q \geqslant n$ and $z \in S(m, q)$, then $\lim _{t \rightarrow \infty}\left(I-G_{q}\right)^{t} z=z-A$ - $M_{q-n}^{-1} A z$.

From the proof of Theorem 3, it follows that if $z$ is of the form $A \cdot w$ for some $w \in S(m, q)$, then $\lim _{t \rightarrow \infty}\left(I-G_{q}\right)^{t} z=0$. Now if $A z=0,\left(I-G_{q}\right)^{t} z$ z. But any $z$ in $S(m, q)$ may be written $z=z_{1}+z_{2}, z_{1}=z-A \cdot M_{q-n}^{-1} A z$, $z_{2}=A \cdot M_{q-n}^{-1} A z$. Then $\lim _{t \rightarrow \infty}\left(I-G_{q}\right)^{t} z_{2}=0$ and $\lim _{t \rightarrow \infty}\left(I-G_{q}\right)^{t} z_{1}^{q-n}=z_{1}$ since $A z_{1}=0$.

LemMa 5. Suppose $q \in Z^{+}, q \geqslant n, \eta$ is a continuous function from $[0, \infty)$ to $S(m, q)$ and $\lim _{t \rightarrow \infty} \eta(t)=\phi \in S(m, q)$. Then $\int_{0}^{\lambda} d\left(I-G_{q}\right)^{\lambda-j} \eta=A$ - $M_{q-n}^{-1} A \phi$.

This follows from Lemma 4 using the fact that $\int_{0}^{\lambda} d\left(I-G_{q}\right)^{\lambda-j}=I$ $-\left(I-G_{q}\right)^{\lambda}$.

LEMMA 6. Suppose $0<\beta, \gamma \leqslant \infty$ and $\phi$ is a continuous function from $[0, \beta) \times[0, \gamma)$ to $[0, \infty)$. Then if $M>0$, there is $L>0$ such that if $\mu$ is a nonnegative continuous real-valued function on $\left[0, L^{\prime}\right]$ for some $L^{\prime}$ in $(0, L]$ and $\mu(x) \leqslant \phi(x, x \mu(x))$ for all $x \in\left[0, L^{\prime}\right]$, then $\mu(x)<\phi(0,0)+M$ for all $x$ $\in\left[0, L^{\prime}\right]$.

The assumption of the negation of this lemma leads easily to a contradiction.

4. Proof of main results.

Proof of Theorem 4. Suppose $U \in H(\alpha), k \in Z^{+}, t_{i}>0, i=1, \ldots, k$. By induction,

$$
\begin{aligned}
J\left(t_{k}\right) \cdots J\left(t_{1}\right) U= & L\left(t_{k}\right) \cdots L\left(t_{1}\right) U \\
& +L\left(t_{k}\right) \cdots L\left(t_{2}\right)\left(I-L\left(t_{2}\right)\right) B f_{J\left(t_{1}\right) U} \\
& +\cdots+L\left(t_{k}\right)\left(I-L\left(t_{k-1}\right)\right) B f_{J\left(t_{k-1}\right) \cdots J\left(t_{1}\right) U} \\
& +\left(I-L\left(t_{k}\right)\right) B_{J\left(t_{k}\right) \cdots J\left(t_{1}\right) U}
\end{aligned}
$$


For each positive integer $q$ denote by $H_{q}$ the following hypothesis:

There is a unique continuous function $w_{q}$ from $[0, \infty)$ to $S(m, q)$ so that if $\varepsilon, \lambda_{0}>0$, there is $\delta>0$ so that if $0 \leqslant \lambda \leqslant \lambda_{0}, k \in Z^{+}, \delta \geqslant t_{i} \geqslant 0, i=1$, $\ldots, k$ and $t_{1}+\cdots+t_{k}=\lambda$, then

$$
\left\|\left(J\left(t_{k}\right) \cdots J\left(t_{1}\right) U\right)^{(q)}(0)-w_{q}(\lambda)\right\|<\varepsilon .
$$

Each of $H_{1}, \ldots, H_{n-1}$ are true since $(J(t) U)^{(q)}(0)=U^{(q)}(0), q=0,1$, $\ldots, n-1, t>0$. Hence $w_{q}(\lambda)=U^{(q)}(0), q=0,1, \ldots, n-1, \lambda \geqslant 0$.

Suppose now that $q$ is a positive integer and $H_{1}, \ldots, H_{q-1}$ are true.

A preliminary observation is in order. Define $Z_{q, \lambda} \in H(\lambda)$ so that $Z_{q, \lambda}(x)$ $=\sum_{i=0}^{q-n+h}(1 / i !) w_{i}(\lambda) x^{i}, x \in E_{m}, \lambda \geqslant 0$. Then, if $\varepsilon>0$, there is $\delta>0$ so that if $0 \leqslant \lambda<\lambda_{1}, k \in Z^{+}, 0 \leqslant t_{i} \leqslant \delta, i=1, \ldots, k, t_{1}+\cdots+t_{k}=\lambda$, then

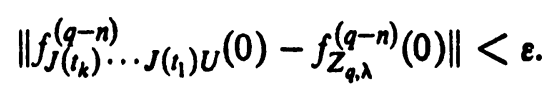

Now for $k$ a positive integer and $t_{i}>0, i=1, \ldots, k$,

$$
\begin{aligned}
\left(J\left(t_{k}\right) \cdots J\left(t_{1}\right) U\right)^{(q)}(0)= & \left(L\left(t_{k}\right) \cdots L\left(t_{1}\right) U\right)^{(q)}(0) \\
& +\left(L\left(t_{k}\right) \cdots L\left(t_{2}\right)\left(I-L\left(t_{1}\right)\right)\right)_{q} A \cdot M_{q-n}^{-1} f_{J}^{(q-n)}(0) U \\
& +\cdots+\left(I-L\left(t_{k}\right)\right)_{q} A \cdot M_{q-n}^{-1} f_{J}^{(q-n)}\left(t_{k}\right) \cdots J\left(t_{1}\right) U U
\end{aligned}
$$

Using the above observation and Lemmas 2 and 3 one obtains:

If $\varepsilon, \lambda_{0}>0$, there is $\delta>0$ such that if $0 \leqslant \lambda \leqslant \lambda_{0}, k \in Z^{+}, \delta>t_{i}>0$, $i=1, \ldots, k, t_{1}+\cdots+t_{k}=\lambda$, then

$$
\begin{aligned}
\|\left(J\left(t_{k}\right)\right. & \left.\cdots J\left(t_{1}\right) U\right)^{(q)}(0) \\
& -\left[\left(I-G_{q}\right)^{\lambda} U^{(q)}(0)+\int_{0}^{\lambda} d\left(I-G_{q}\right)^{\lambda-j}\left(A \cdot M_{q-n}^{-1} f_{Z_{q, J}}^{(q-n)}(0)\right)\right] \|<\varepsilon .
\end{aligned}
$$

This proves $H_{q}$ with

$$
w_{q}(\lambda)=\left(I-G_{q}\right)^{\lambda} U^{(q)}(0)+\int_{0}^{\lambda} d\left(I-G_{q}\right)^{\lambda-j}\left(A \cdot M_{q-n}^{-1} f_{Z_{q, j}}^{(q-n)}(0)\right) .
$$

Hence all of $H_{1}, H_{2} \cdots$ are true.

Suppose $q \geqslant n, \lambda>0$ and $z \in S(m, q)$, then 


$$
\begin{aligned}
\left\|\left(I-\left(I-G_{q}\right)^{\lambda}\right) B_{q} z\right\|^{2} & =\left\|\left(I-\left(I-G_{q}\right)^{\lambda}\right) A \cdot M_{q-n}^{-1} z\right\|^{2} \\
& =\left\langle M_{q-n}^{-1}\left(I-\left(I-G_{q}\right)^{\lambda}\right)^{2} z, z\right\rangle \\
& <\left(\sup _{\varepsilon \in(0,1]} \varepsilon^{-1}\left(1-(1-\varepsilon)^{\lambda}\right)^{2}\right)\|z\|^{2} .
\end{aligned}
$$

The upper bound exists since $\lim _{\varepsilon \rightarrow 0} \varepsilon^{-1}\left(1-(1-\varepsilon)^{\lambda}\right)^{2}=0$. Denote

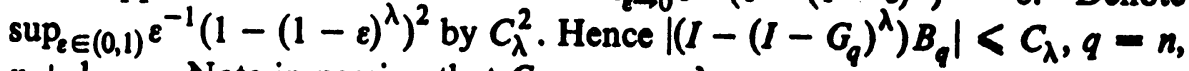
$n+1, \ldots$. Note in passing that $C_{\lambda} \rightarrow \infty$ as $\lambda \rightarrow \infty$.

For $\lambda>0, q>n$, define

$$
\theta_{q, \lambda}(s)=\sum_{i=0}^{q-n+h}(1 / i !)\left(\sup _{t \in[0, \lambda]}\left\|w_{i}(t)\right\|\right) s^{i}
$$

for all $s \geqslant 0$. Note that $\theta_{q, \lambda}^{(i)}(0)=\sup _{t \in[0, \lambda]}\left\|w_{i}(t)\right\|, i=0,1, \ldots, q-n+h$. From the fact that

$$
w_{q}(t)=\left(I-G_{q}\right)^{t} U^{(q)}(0)+\int_{0}^{t} d\left(I-G_{q}\right)^{t-j}\left(A \cdot M_{q-n}^{-1} f_{Z_{N}}^{(q-n)}(0)\right)
$$

for $0 \leqslant t \leqslant \lambda$, it follows that

$$
\left\|w_{q}(t)\right\| \leqslant\left\|U^{(q)}(0)\right\|+C_{\lambda}\left(F\left(j, \theta_{q, \lambda}, \theta_{q, \lambda}^{\prime}, \ldots, \theta_{q, \lambda}^{(h)}\right)\right)^{(q-n)}(0)
$$

for $0 \leqslant t \leqslant \lambda$. As in the proof of Theorem 3, denote by $g$ the function so that

$$
g(t)=\sum_{i=0}^{\infty}(1 / i !)\left\|U^{(i)}(0)\right\| t^{i}, \quad|t|<\rho(U) .
$$

Denote by $\delta$ a small enough positive number so that there is a unique function $y_{\lambda}$ on $(-\delta, \delta)$ so that $\left(y_{\lambda}(0), y_{\lambda}^{\prime}(0), \ldots, y_{\lambda}^{(n-1)}(0)\right)=(\|U(0)\|$, $\left.\left\|U^{\prime}(0)\right\|, \ldots,\left\|U^{(n-1)}(0)\right\|\right)$ and

$$
y_{\lambda}^{(n)}(x)=g(x)+C_{\lambda} F\left(x, y_{\lambda}(x), y_{\lambda}^{\prime}(x), \ldots, y_{\lambda}^{(h)}(x)\right), \quad|x|<\delta,
$$

where $F$ is as in the proof of Theorem 3. It follows by induction that $\sup _{t \in[0, \lambda]}\left\|w_{q}(t)\right\| \leqslant y_{\lambda}^{(q)}(0), q=0,1,2, \ldots$ Since there is a positive number $\delta^{\prime}$ and a number $m_{0}$ so that

$$
\sum_{i=0}^{\infty}(1 / i !) y_{\lambda}^{(i)}(0) s^{i}<m_{0} \text { if } 0 \leqslant s \leqslant \delta^{\prime}
$$

it follows that

$$
\sum_{i=0}^{\infty}(1 / i !)\left\|w_{i}(t)\right\| s^{i} \leqslant m_{0}, \quad 0 \leqslant s \leqslant \delta^{\prime}, \quad 0 \leqslant t \leqslant \lambda .
$$


Define $(K(\lambda) U)(x)$ as the member of $H(\alpha)$ so that

$$
(K(\lambda) U)(x)=\sum_{i=0}^{\infty}(1 / i !) w_{i}(\lambda) x^{i}, \quad\|x\|<\delta^{\prime} .
$$

For $\lambda>0, q \in Z^{+}$denote

$\sup \left\{\left\|\left(J\left(t_{k}\right) \cdots J\left(t_{1}\right) U\right)^{(q)}(0)\right\| \mid k \in Z^{+}, t_{i} \geqslant 0, i=1, \ldots, k\right.$

$$
\left.t_{1}+\cdots+t_{k} \leqslant \lambda\right\} \text { by } X_{g}(\lambda) \text {. }
$$

From the formula for $\left(J\left(t_{k}\right) \cdots J\left(t_{1}\right) U\right)^{(k)}(0): X_{g}(\lambda) \leqslant\left\|U^{(q)}(0)\right\|$ $+C_{\lambda}\left(F\left(j, \eta_{q}, \eta_{q}^{\prime}, \ldots, \eta_{q}^{(h)}\right)\right)^{(q-n)}(0)$ where $\eta_{q, \lambda}(s)=\sum_{i=0}^{q-n+h}(1 / i !) X_{i}(\lambda) s^{i}$, all $s>0$. For $y$ as above, it follows by induction that $X_{q}(\lambda) \leqslant y_{\lambda}^{(q)}(0), q=0,1$, $2, \ldots$.

Hence the following is true: If $\lambda_{0}>0, \varepsilon>0$ there are $\delta^{\prime}, \delta>0$ such that if $0 \leqslant \lambda \leqslant \lambda_{0}, k \in Z^{+}, \delta \geqslant t_{i} \geqslant 0, i=1, \ldots, k, t_{1}+\cdots+t_{k}=\lambda, 0<s$ $<\delta^{\prime}$, then

$$
\sum_{q=0}^{\infty}(1 / q !)\left\|\left(J\left(t_{k}\right) \cdots J\left(t_{1}\right) U\right)^{(q)}(0)-(K(\lambda) U)^{(q)}(0)\right\| s^{q}<\varepsilon .
$$

A special case of this is the fact that if $\lambda>0$, then $(J(\lambda / n))^{n} U$ converges to $K(\lambda) U \in H(\alpha)$ as $n \rightarrow \infty$, the first conclusion to Theorem 4 .

The second conclusion is shown by induction. From the first part of the argument, if $U \in H(\alpha)$,

$$
(K(\lambda) U)^{(q)}(0)=\left(I-G_{q}\right)^{\lambda} U^{(q)}(0)+\int_{0}^{\lambda} d\left(I-G_{q}\right)^{\lambda-j}\left(A \cdot M_{q-n}^{-1} f_{K(j) U}^{(q-n)}(0)\right)
$$

$\lambda \geqslant 0, q=n, n+1, \ldots$. Note that if $\beta, \gamma>0$, then $(K(\beta) K(\gamma) U)^{(q)}(0)$ $=U^{(q)}(0)=(K(\beta+\gamma) U)^{(q)}(0), q=0,1, \ldots, n-1$ so

$$
f_{K(\beta) K(\gamma) U}^{(0)}(0)=f_{K(\beta+\gamma) U}^{(0)}(0) \text {. }
$$

Suppose that $q \in Z^{+}$and $(K(\beta) K(\gamma) U)^{(i)}(0)=(K(\beta+\gamma) U)^{(i)}(0), i$ $=0,1, \ldots, q-1, \beta, \gamma>0$. Then $f_{K(\beta) K(\gamma) U}^{(q-n)}(0)=f_{K(\beta+\gamma) U}^{(q-n)}(0), \beta, \gamma \geqslant 0$. Hence

$$
\begin{aligned}
(K(\beta) K(\gamma) U)^{(q)}(0)= & (K(\beta)(K(\gamma) U))^{(q)}(0) \\
= & \left(I-G_{q}\right)^{\beta}(K(\gamma) U)^{(q)}(0) \\
& +\int_{0}^{\beta} d\left(I-G_{q}\right)^{\beta-j}\left(A \cdot M_{q-n}^{-1} f_{K(j) K(\gamma) U}^{(q-n)}(0)\right)
\end{aligned}
$$




$$
\begin{aligned}
= & \left(I-G_{q}\right)^{\beta}\left[\left(I-G_{q}\right)^{\gamma} U^{(q)}(0)+\int_{0}^{\gamma} d\left(I-G_{q}\right)^{\gamma-1}\left(I \cdot M_{q-n}^{-1} f_{K}^{(q-n) U}(0)\right)\right] \\
& +\int_{0}^{\beta} d\left(I-G_{q}\right)^{\beta-j} A \cdot M_{q-n}^{-1} f_{K}^{(q-n)}(j+\gamma) U(0) \\
= & \left(I-G_{q}\right)^{\beta+\gamma} U^{(q)}+\int_{0}^{\gamma} d\left(I-G_{q}\right)^{\gamma+\beta-j}\left(A \cdot M_{q-n}^{-1} f_{K}^{(q-n) U}(0)\right) \\
& +\int_{\gamma}^{\beta+\gamma} d\left(I-G_{q}\right)^{\gamma+\beta-j}\left(A \cdot M_{q-n}^{-1} f_{K}^{(q-n) U}(0)\right) \\
= & (K(\beta+\gamma) U)^{(q)}(0), \quad \beta, \gamma>0 .
\end{aligned}
$$

Hence $K(\beta+\gamma) U=K(\beta) K(\gamma) U$ ior all $\beta, \gamma>0, U \in H(\alpha)$.

Proof of Theorem 5. Suppose $J \in H(\alpha)$. To show (a) is true, recall that if $\lambda>0,(K(\lambda) U)^{(q)}(0)=U^{(q)}(0), q=0,1, \ldots, n-1$, and $(K(\lambda) U)^{(q)}(0)$

$$
\begin{aligned}
& =\left(I-G_{q}\right)^{\lambda} U^{(q)}(0)+\int_{0}^{\lambda} d\left(I-G_{q}\right)^{\lambda-j}\left(A \cdot M_{q-n}^{-1} f_{K}^{(q-n) U}(0)\right) \\
& =\left(I-G_{q}\right)^{\lambda} U^{(q)}(0)+A \cdot M_{q-n}^{-1} \int_{0}^{\lambda} d\left(I-M_{q-n}\right)^{\lambda-j}\left(f_{K}^{(q-n) U}(0)\right),
\end{aligned}
$$

$q=n, n+1, \ldots$ Using Lemmas 3,4 , and 5 one has inductively that if $r_{i}=U^{(i)}(0), i=0,1, \ldots, n-1$ and $q \in Z^{+}, q>n$, then

$$
\begin{aligned}
& \lim _{\lambda \rightarrow \infty}(K(\lambda) U)^{(q)}(0)= U^{(q)}(0)-A \cdot M_{q-n}^{-1} A U^{(q)}(0) \\
&+A \cdot M_{q-n}^{-1} A\left(A \cdot M_{q-n}^{-1} f_{Z_{q}}^{(q-n)}(0)\right) \\
&=U^{(q)}(0)-A \cdot M_{q-n}^{-1} A U^{(q)}(0)+A \cdot M_{q-n}^{-1} f_{Z_{q}}^{(q-n)}(0)=r_{q}
\end{aligned}
$$

where $Z_{q}(x)=\sum_{i=0}^{q-1}(1 / i !) r_{i} x^{i}$ for all $x \in E_{m}$.

For $q \in Z^{+}, q>n, \lambda>0$,

$$
\begin{aligned}
A\left((K(\lambda) U)^{(q)}(0)\right)= & A\left(\left(I-G_{q}\right)^{\lambda} U\right)^{(q)}(0) \\
& +\int_{0}^{\lambda} d\left(I-M_{q-n}\right)^{\lambda-j}\left(f_{K(j) U}^{(q-n)}(0)\right) .
\end{aligned}
$$

Define $W$ on $[0, \infty)$ so that $W(\lambda)=A\left((K(\lambda) U)^{(n)}\right), \lambda>0$, and note that if $q \in Z^{+}, q>n$, then

$$
\begin{aligned}
(K(t) U)^{(q)}(0)= & {\left[(K(t) U)^{(q)}(0)-A \cdot M_{q-n}^{-1} A\left((K(t) U)^{(q)}(0)\right)\right] } \\
& +A \cdot M_{q-n}^{-1} A\left((K(t) U)^{(q)}(0)\right) \\
= & Z^{(q)}(0)+B q\left(W(t)^{(q-n)}(0)\right), \quad t \geqslant 0
\end{aligned}
$$


where $Z \in H(\alpha)$ so that $Z^{(q)}(0)=U^{(q)}(0), q=0,1, \ldots, n-1, Z^{(q)}(0)$ $=U^{(q)}(0)-A \cdot M_{q-n}^{-1} A U^{(q)}(0), q=n, n+1, \ldots$ Use was made of the following important fact: If $t>0$, then

$$
\begin{aligned}
& (K(t) U)^{(q)}(0)=\left(I-G_{q}\right)^{t} U^{(q)}(0)+A \cdot M_{q-n}^{-1} \theta, \\
\theta= & \int_{0}^{t} d\left(I-M_{q-n}\right)^{t-j}\left(f_{K(j) U}^{(q-n)}(0)\right),(K(t) U)^{(q)}(0) \\
& -A \cdot M_{q-n}^{-1}\left(A(K(t) U)^{(q)}(0)\right) \\
= & \left(I-G_{q}\right)^{t} U^{(q)}(0)+A \cdot M_{q-n}^{-1} \theta \\
& -A \cdot M_{q-n}^{-1} A\left(I-G_{q}\right)^{t} U^{(q)}(0)-A \cdot M_{q-n}^{-1} A\left(A \cdot M_{q-n}^{-1} \theta\right) \\
= & \left(I-G_{q}\right)^{t} U^{(q)}(0)-A \cdot M_{q-n}^{-1} A\left(I-G_{q}\right)^{t} U^{(q)}(0) \\
= & \left(I-G_{q}\right)^{t}\left[U^{(q)}(0)-A \cdot M_{q-n}^{-1} A U^{(q)}(0)\right] \\
= & \left(I-G_{q}\right)^{t} \lim _{s \rightarrow \infty}\left(I-G_{q}\right)^{s} U^{(q)}(0) \\
= & \lim _{s \rightarrow \infty}\left(I-G_{q}\right)^{s+t} U^{(q)}(0) \\
= & \lim _{s \rightarrow \infty}\left(I-G_{q}\right)^{s} U^{(q)}(0)=U^{(q)}(0)-A \cdot M_{q-n}^{-1} A U^{(q)}(0)=Z^{(q)}(0)
\end{aligned}
$$

using Lemma 4. Hence

$$
(K(t) U)^{(q)}(0)=Z^{(q)}(0)+B_{q} W(t)^{(q \cdot-n)}(0),
$$

i.e. $K(t) U=Z+B W(t), t>0$. Therefore,

$$
\begin{aligned}
(W(\lambda))^{(q-n)}(0)= & \left(I-M_{q-n}\right)^{\lambda}\left(A U^{(q)}(0)\right) \\
& +\int_{0}^{\lambda} d\left(I-M_{q-n}\right)^{\lambda-j}\left(f_{Z+B W(j)}^{(q-n)}(0)\right),
\end{aligned}
$$

$q=n, n+1, \ldots, \lambda \geqslant 0$.

Define $\eta \in H$ so that $\eta^{(q-n)}(0)=A U^{(q)}(0), q=n, n+1, \ldots$. Suppose $s>0$. Then

$$
\left\|W(s)^{(q)}(0)\right\|<\left\|\eta^{(q)}(0)\right\|+\sup _{0<i<s}\left\|f_{Z+B W(t)}^{(q)}(0)\right\|
$$

$q=0,1,2, \ldots$, so that if $\lambda>0$, 


$$
\sup _{0<s<\lambda}\left\|W(s)^{(q)}(0)\right\|<\left\|\eta^{(q)}(0)\right\|+\sup _{0<s<\lambda} \sup _{0<t<s}\left\|f_{Z+B W(t)}^{(q)}(0)\right\|,
$$

i.e.,

$$
\sup _{0<s<\lambda}\left\|W(s)^{(q)}(0)\right\|<\left\|\eta^{(q)}(0)\right\|+\sup _{0<s<\lambda}\left\|f_{Z+B W(s)}^{(q)}(0)\right\| .
$$

If $g \in H, 0<t<\rho(g)$, then $\|g\|_{t}$ denotes $\sum_{i=0}^{\infty}(1 / i !)\left\|g^{(i)}(0)\right\| t^{i}$.

Denote by $F$ the function which relates to $f$ as in Lemma 1 . Then for any $g \in H, q \in Z^{+}, q \geqslant n,\left\|f_{Z+B_{g}(0)}^{(q)}\right\|<\left(F\left(\Phi_{g}\right)\right)^{(q)}(0)$ where $\Phi=\left(\Phi_{g, 1}, \ldots\right.$, $\left.\Phi_{g, h+2}\right)$ and for $i=2, \ldots, h+2$,

$$
\begin{gathered}
\Phi_{g, i}=\left\|(Z+B g)^{(i-2)}\right\|_{t} \leqslant\left\|Z^{(i-2)}\right\|_{t}+\left\|(B g)^{(i-2)}\right\|_{t} \\
=\left\|Z^{(i-2)}\right\|_{t}+\sum_{r=n}^{\infty}\left(\frac{1}{(r-i+2) !}\right)\left\|A \cdot M_{r-n}^{-1} g^{(r-n)}(0)\right\| t^{r-i+2} \\
<\left\|Z^{(i-2)}\right\|_{t}+t^{n-i+2} \sum_{r=n}^{\infty}\left(\frac{1}{(r-n) !}\right)\left(\left(\begin{array}{l}
r \\
n
\end{array}\right)^{1 / 2} /((r-i+2) \cdots(r-n+1))\right) \\
\left\|g^{(r-n)}(0)\right\| t^{r-n}<\left\|Z^{(i-2)}\right\|_{t}+C t^{n-i+2}\left\|_{g}\right\|_{t}, \quad 0<t<\rho(Z), \rho(g)
\end{gathered}
$$

where $C$ is a number so that

$$
C>\left(\begin{array}{l}
q \\
n
\end{array}\right)^{1 / 2} /((q-i+2) \cdots(q-n+1)), \quad q=n, n+1, \ldots,
$$

(see $[5,85])$. In the above the fact was used that if $w \in S(m, r-n)$, then

$$
\left\|A \cdot M_{r-n}^{-1} w\right\|^{2} \leqslant\left\langle M_{r-n}^{-1} w, w\right\rangle \leqslant\left(\begin{array}{l}
r \\
n
\end{array}\right)\|w\|^{2}, \quad(\text { see [4]). }
$$

Take $\Phi_{1, q}(t)=t$, for all numbers $t$. Hence,

$$
\begin{aligned}
\left\|f_{Z+B g}\right\|_{t} & =\sum_{q=0}^{\infty}(1 / q !)\left\|f_{Z+B g}^{(q)}(0)\right\| t^{q} \\
& <\sum_{q=0}^{\infty}(1 / q !)\left(F\left(\Phi_{g}\right)\right)^{(q)}(0) t \\
& =\left(F\left(\Phi_{g}\right)\right)(t), \quad 0<t<\rho(Z), \rho(g) .
\end{aligned}
$$

Suppose $\lambda>0$. Define $C_{\lambda}(t)=\sum_{q=0}^{\infty}(1 / q !) \sup _{0<s<\lambda}\left\|W(s)^{(q)}(0)\right\| t^{q}$ for all $t$ for which this converges (that it converges for some $t>0$ follows from the argument for Theorem 4).

Note that $\left\|f_{Z+B W(t)}(0)\right\| \leqslant\left(F\left(\Phi_{W(t)}\right)\right)^{(q)}(0) \leqslant(F(\Phi))^{(q)}(0)$ where $\Phi$ $=\left(\Phi_{1}, \ldots, \Phi_{h+2}\right), \Phi_{i}(t) \equiv\left\|Z^{(i-2)}\right\|_{t}+C t^{n-i+2} C_{\lambda}(t), 0 \leqslant t<\rho\left(C_{\lambda}\right), \rho\left(Z^{(i-2)}\right)$, 
$i=2, \ldots, h+1, \Phi_{1}(t)=t$, all $t$. From the fact that

$$
\sup _{0<s<\lambda}\left\|W(s)^{(q)}(0)\right\| \leqslant\left\|\eta^{(q)}(0)\right\|+\sup _{0<s<\lambda}\left\|F_{Z+B W(s)}^{(q)}(0)\right\|,
$$

it follows that

$$
\sup _{0<s<\lambda}\left\|W(s)^{(q)}(0)\right\| \leqslant\left\|\eta^{(q)}(0)\right\|+(F(\Phi))^{(q)}(0)
$$

and so

$$
\begin{aligned}
C_{\lambda}(t)= & \sum_{q=0}^{\infty}(1 / q !) \sup _{0<s<\lambda}\left\|W(s)^{(q)}(0)\right\| t^{q} \\
& <\|\eta\|_{t}+\sum_{q=0}^{\infty}(1 / q !) F(\Phi)^{(q)}(0) t^{q} \\
& =\|\eta\|_{t}+(F(\Phi))(t) \\
& =\|\eta\|_{t}+F\left(t, \Phi_{2}(t), \ldots, \Phi_{n+2}(t)\right) \\
& =\|\eta\|_{t}+F\left(t,\|Z\|_{t}+C t^{n} C_{\lambda}(t),\left\|Z^{\prime}\right\|_{t}+C t^{n-1} C_{\lambda}(t), \cdots,\right. \\
& \left.\left\|Z^{(h)}\right\|_{t}+C t^{n-h} C_{\lambda}(t)\right), \\
& \quad 0<t<\rho(F(\Phi)) .
\end{aligned}
$$

Denote by $\beta, \gamma$ two positive numbers so that if $D\left(t, x_{0}, x_{1}, \ldots, x_{h}\right)=\left(t,\|Z\|_{t}\right.$ $\left.+t^{n-1} x_{0},\left\|Z^{\prime}\right\|_{t}+t^{n-2} x_{1}, \ldots,\left\|Z^{(h)}\right\|_{t}+t^{n-h-1} x_{h}\right), 0<t<\beta, 0 \leqslant x_{i}<\gamma, i$ $=0,1, \ldots, h$, then $\sum_{q=0}^{\infty}(1 / q !)(F(D))^{(q)}(0) t^{q}$ converges. Define

$$
\begin{aligned}
\phi(t, x)=\|\eta\|_{t}+F\left(t,\|Z\|_{t}+t^{n-1} x,\left\|Z^{\prime}\right\|_{t}+t^{n-2} x, \cdots,\right. & \left.\left\|Z^{(h)}\right\|_{t}+t^{n-h-1} x\right), \\
& 0 \leqslant t<\beta, 0 \leqslant x<\gamma .
\end{aligned}
$$

By Lemma 6, there is $L>0$ so that if $0<L^{\prime} \leqslant L$ and $\mu$ is a nonnegative continuous function on $\left[0, L^{\prime}\right]$ so that $\mu(t) \leqslant \phi(t, t \mu(t))$ for all $t \in\left[0, L^{\prime}\right]$, then $\mu(t)<\phi(0,0)+1, t \in\left[0, L^{\prime}\right]$.

Suppose $\lambda>0$. Then $C_{\lambda}(t) \leqslant \phi\left(t, t C_{\lambda}(t)\right)$ for all $t$ in some interval $\left[0, L^{\prime}\right], 0$ $<L^{\prime} \leqslant L$. Hence $C_{\lambda}(t)<\phi(0,0)+1$ for $t \in\left[0, L^{\prime}\right]$. Since $C_{\lambda}$ is increasing and continuous on its domain (all $t \geqslant 0$ such that its power series about 0 converges), the assumption that $C_{\lambda}$ does not have the property that its domain includes $[0, L)$ and $C_{\lambda}(t) \leqslant \phi(0,0)+1$ for all $t \in[0, L]$ leads to a contradiction. This gives $\|W(\lambda)\|_{t} \leqslant \phi(0,0)+1 \equiv m_{0}$ for all $\lambda \geqslant 0, t \in[0, L]$. This implies that there is $m^{\prime}>0$,

$$
\|K(\lambda) U\|_{t} \leqslant m^{\prime}, \quad \lambda \geqslant 0, \quad 0 \leqslant t \leqslant L_{0}, \rho(U) .
$$


This together with the fact that $(K(\lambda) U)^{(q)}(0)$ converges as $\lambda \rightarrow \infty, q=0,1$, 2 , $\therefore$, gives that there is $Y \in H(\alpha)$ so that $K(\lambda) U \rightarrow Y$ as $\lambda \rightarrow \infty$. This concludes a proof to part (a) of the theorem.

Note that $Y^{(q)}(0)=r_{q}, q=0,1,2, \ldots$, where $r_{0}, r_{1}, r_{2}, \ldots$ are defined inductively early in the proof of this theorem.

Now part (b) is demonstrated.

For $Z \in H(\alpha)$, define $Q Z \in H(\alpha)$ so that

$$
(Q Z)(x)=\sum_{q=0}^{n-1}\left(\frac{1}{q !}\right) Z^{(q)}(0) x^{q}+\sum_{q=n}^{\infty}\left(\frac{1}{q !}\right)\left(\left(I-G_{q}\right) Z^{(q)}(0)\right) x^{q},
$$

$\|x\|<\rho(Z)$

and for $Z \in H$, define

$$
(V Z)(x)=\sum_{q=n}^{\infty}\left(\frac{1}{q !}\right)\left(A \cdot Z^{(q-n)}(0)\right) x^{q}, \quad\|x\|<\rho(Z) .
$$

Then $T Z=Q Z+V f_{Z}, Z \in H(\alpha)$. By induction,

$$
T^{k} U=Q^{k} U+\sum_{i=0}^{k-1} Q^{l} V f_{T^{k-1-1} U}, \quad k=1,2, \ldots
$$

Define $W_{k}=A\left(T^{k} U\right)^{(n)}, k=1,2, \ldots$ Then for $k, q \in Z^{+}, q>n$,

$$
\begin{aligned}
\left(T^{k} U\right)^{(q)}(0) & =\left(I-G_{q}\right)^{k} U^{(q)}(0)+\sum_{i=0}^{k-1}\left(I-G_{q}\right)^{i} A \cdot f_{T^{(q-1-1}}^{(q-n)} U(0) \\
& =\left(I-G_{q}\right)^{k} U^{(q)}(0)+\sum_{i=0}^{k-1} A \cdot\left(I-M_{q-n}\right)^{l} f_{T}^{(q-n)}(0) U
\end{aligned}
$$

and so

$$
W_{k}^{(q-n)}(0)=\left(I-M_{q-n}\right)^{k} A U^{(q)}(0)+\sum_{i=0}^{k-1} M_{q-n}\left(I-M_{q-n}\right)^{i} f_{T}^{(q-n)}(0-1-1 U(0) .
$$

As in part (a),

$$
T^{k} U=Z+B W_{k}
$$

Since $\sum_{i=0}^{k-1} M_{q-n}\left(I-M_{q-n}\right)^{i}=I-M_{q-n}^{k}$ and $0<I-M_{q-n}^{k}<I, k=1,2$, $\ldots$, it follows that

$$
\left\|W_{k}^{(q-n)}(0)\right\| \leqslant\left\|A U^{(q)}(0)\right\|+\sup _{i=1, \ldots, k-1}\left\|f_{T^{k-1-1}}^{(q-n)} U(0)\right\|, \quad q=n, n+1, \ldots
$$

and hence 


$$
\left\|W_{k}^{(q)}(0)\right\| \leqslant\left\|\eta^{(q)}(0)\right\|+\sup _{i=1, \ldots, k-1}\left\|f_{Z+B W_{i}}^{(q)}(0)\right\|
$$

$q=0,1,2, \ldots, k=1,2, \ldots$, with $\eta$ as in part (a).

That there are $m_{0}, L_{0}>0$ so that $\left\|W_{k}\right\|_{t} \leqslant m_{0}, k=1,2, \ldots, t \in\left[0, L_{0}\right]$ is proved essentially as the corresponding fact in part (a). Similarly, there is $m^{\prime}>0$ so that $\left\|T^{k} U\right\|_{t}<m^{\prime}, 0 \leqslant t \leqslant L_{0}, \rho(U)$.

Observe that

$$
\begin{aligned}
\left(T^{k} U\right)^{(q)}(0) & =\left(I-G_{q}\right)^{k} U^{(q)}(0)+\sum_{i=0}^{k-1}\left(I-G_{q}\right)^{i} A \cdot f_{T^{k-1-1}}^{(q-n)}(0) \\
& =\left(I-G_{q}\right)^{k} U^{(q)}(0)+\sum_{i=0}^{k-1} A \cdot\left(I-M_{q-n}\right)^{l} f_{T^{k-1-1}}^{(q-n)}(0) \\
& \rightarrow U^{(q)}(0)-A \cdot M_{q-n}^{-1} A U^{(q)}(0)+A \cdot M_{q-n}^{-1} f_{Y}^{(q-n)}(0)
\end{aligned}
$$

as $k \rightarrow \infty$, inductively on $q$, since

$$
\begin{aligned}
\sum_{i=0}^{k-1}\left(I-M_{q-n}\right)^{i}=M_{q-n}^{-1}-M_{q-n}^{-1}\left(I-M_{q-n}\right)^{k} \rightarrow M_{q-n}^{-1} \text { as } k & \rightarrow \infty, \\
q & =n, n+1, \ldots .
\end{aligned}
$$

This convergence, together with the boundedness of $\left\{\left\|T^{k} U\right\|_{\ell}\right\}_{k=1}^{\infty}$ for some $t>0$, gives that part (b) is true. Note that part (b) implies Theorem 1.

Now to demonstrate part (c). For $\lambda>0$,

$$
J(\lambda) U=L(\lambda) U+(I-L(\lambda)) B f_{J(\lambda) U} .
$$

Hence for each $q \in Z^{+},(J(\lambda) U)^{(q)}(0)=L(\lambda)_{q} U^{(q)}(0)+\left(I-L(\lambda)_{q}\right) A$ $\cdot M_{q-n}^{-1} f_{J}^{(q) U}(0)$ and

$$
\left\|A(J(\lambda) U)^{(q)}(0)\right\| \leqslant\left\|A U^{(q)}(0)\right\|+\left\|f_{J}^{(q-n) U}(0)\right\| .
$$

Define $W(\lambda)=A(J(\lambda) U)^{(n)}$ and take $\eta$ and $Z$ as in parts (a) and (b). Then

$$
\left\|W(\lambda)^{(q)}(0)\right\| \leqslant\left\|\eta^{(q)}\right\|+\left\|f_{Z+B W(\lambda)}^{(q)}(0)\right\| .
$$

By an argument similar to those in parts (a) and (b) (but simpler since one has $\left\|f_{Z+B W(\lambda)}^{(q)}(0)\right\|$ on the right-hand side instead of $\sup _{0<x<\lambda}\left\|f_{Z+B W(x)}^{(q)}(0)\right\|$ in part (a) or $\sup _{i=1, \ldots, k-1}\left\|f_{Z+B W}^{(q)}(0)\right\|$ in part (b)) one arrives at the fact that there is $L_{0}, m_{0}>0$ so that $\|W(\lambda)\|_{t} \leqslant m_{0}, 0 \leqslant t \leqslant L_{0}, \lambda \geqslant 0$. This leads to the existence of $t>0$ so that $\left\{\|J(\lambda) U\|_{t}\right\}_{\lambda \geq 0}$ is bounded. Since 


$$
L(\lambda)_{q} U^{(q)}(0) \rightarrow U^{(q)}(0)-A \cdot M_{q-n}^{-1} U^{(q)}(\downarrow) \text { as } \lambda \rightarrow \infty
$$

and

$$
\begin{aligned}
\left(I-L(\lambda)_{q}\right) A \cdot M_{q-n}^{-1} r \rightarrow A \cdot M_{q-n}^{-1} A\left(A \cdot M_{q-n}^{-1} r\right) & =A \cdot M_{q-n}^{-1} r \\
& \text { as } \lambda \rightarrow \infty, \text { r } F S(m, q-n),
\end{aligned}
$$

it follows by induction on $q$ that $(J(\lambda) U)^{(q)}(0) \rightarrow U^{(q)}(0)-A$ - $M_{q-n}^{-1} A U^{(q)}(0)+A \cdot M_{q-n}^{-1} f_{Y}^{(q-n)}$ as $\lambda \rightarrow \infty, q=n, n+1, \ldots$ This, together with the boundedness of $\left\{\|J(\lambda) U\|_{l}\right\}_{\lambda>0}$, gives part (c).

5. Epilogue. Note that if $U \in H(\alpha)$, then $Y$ of Theorem 5 has the f.llowing property:

$Y^{(q)}(0)=U^{(q)}(0), q=0,1, \ldots, n-1$, and $Y^{(n)}(0), Y^{(n+1)}(0), \ldots$ can be defined inductively as follows:

If $q \in Z^{+}, q \geqslant n$, then $Y^{(q)}(0)$ is the element $w$ of $S(m, q)$ satisfying: $\left\|U^{(q)}(0)-w\right\|$ is minimum over all $w \in S(m, q)$ so that $A w=f_{Y_{q-1}}^{(q-n)}(0)$ where

$$
Y_{q-1}(x)=\sum_{i=0}^{n-1}(1 / i !) U^{(i)}(0) x^{i}+\sum_{i=n}^{q-1}(1 / i !) Y^{(i)}(0) x^{i}, \quad x \in E_{m} .
$$

The problem of solving for $w \in S(m, q)$ such that $A w=f_{Y_{q-1}}^{(q-n)}(0)$ is essentially an underdetermined consistent linear system. In the CauchyKowalewski method, noncharacteristic initial conditions are used to add equations so that the resulting linear system has a unique solution. In the present method the minimization breaks the impasse concerning the underdetermined system.

This allows $Y$ to take on some of the character of $U$ in a manner natural to the problem at hand. It is expected (and has beer pointed out in examples in [3] and [5]) that a study of the correspondence: $U \rightarrow Y$ will yield in some instances appropriate (and perhaps new) boundary conditions in a general sense.

It is mentioned in closing that the present point of view seems to apply in substantial instances to systems of nonlinear equations and to more fully nonlinear problems: problems of finding $U$ so that

$$
f\left(x, U(x), U^{\prime}(x), \ldots, U^{(n)}(x)\right)=0
$$

for all $x$ in some region of $E_{m}, f$ from $E_{m} \times R \times S(m, 1) \times \cdots \times S(m, n)$ to $R$. Formal power series solutions of many such equations are in hand but the convergence problems seem to this writer to be particularly difficult. 


\section{REFERENCES}

1. H. Brézis and A. Pazy, Semigroups of nonlinear contractions on convex sets, J. Functional Analysis 6 (1970), 237-281.

2. M. G. Crandall and T. M. Liggett, $A$ theorem and a counterexample in the theory of semigroups of nonlinear transformations, Trans. Amer. Math. Soc. 160 (1971), 263-278. MR 46 \#750.

3. J. W. Neuberger, Tensor products and successive approximations for partial differential equations, Israel J. Math. 6 (1968), 121-132. MR 38 \#5404.

4. - Norm of symmetric product compared with norm of tensor product, J. Linear Multilinear Algebra 2 (1974), 115-121.

5. - An iterative method for solving nonlinear partial differential equations, Advances in Math. 19 (1976), 245-265.

6. F. Nevanlinna and R. Nevanlinna, Absolute analysis, Die Grundlehren der math. Wiss., Springer-Verlag, Berlin, 1959. MR 22 \# 12176.

Department of Mathematics, Emory University, Atlanta, Georgia 30322 